\title{
Interaktive Karte: DEGUM-zertifizierte Ärztinnen und Ärzte sowie Abteilungen und Zentren finden
}

Wer einen qualifizierten Ultraschalluntersucher sucht, der steht häufig vor einem Problem. Die DEGUM bietet dafür eine Liste aller Ärzt*innen in Deutschland an, die eine Ultraschall-Qualifikation durch eine entsprechende DEGUM-Zertifizierung besitzen.

Wem also zum Beispiel zur Abklärung einer Erkrankung oder zur Ursachensuche von Beschwerden eine Ultraschall-Untersuchung empfohlen wurde oder wer eine zweite ärztliche Meinung zu einem Ultraschallbefund haben möchte, findet mithilfe der DEGUM ganz leicht einen zertifizierten Arzt oder eine Ärztin des gewünschten Fachgebiets in seiner Nähe. Das gilt nicht nur für Patient*innen, sondern auch für Mediziner*innen, die an einer kompetenten sonografischen Aus- oder Fortbildung im eigenen Fachgebiet interessiert sind. Mithilfe der DEGUM können sie deutschlandweit nach zertifizierten Ausbildern und Ausbildungszentren suchen.
Auf der Webseite der DEGUM können alle Interessierten auf einer interaktiven Karte ganz einfach passende Expert*innen finden. Unter www.degum.de/service/zertifizierteaerzte.html lässt sich die Recherche nach Fachgebiet, Postleitzahl und/oder DEGUMStufe filtern.

In der DEGUM können sich sowohl Ärzt*innen als auch Abteilungen und Zentren zertifizieren lassen. So bieten die Sektionen und Arbeitskreise der DEGUM für UltraschallAnwender ein mehrstufiges Zertifizierungssystem, um damit eine flächendeckende und in ihrer Qualität gesicherte UltraschallDiagnostik zu gewährleisten. Das Zertifizierungssystem dient zudem einer spezialisierten Diagnostik in Kliniken und Praxen und dem Erwerb einer spezialisierten, wissenschaftlich orientierten Fachkompetenz für besondere Fragestellungen. Die aktuelle Ultraschallkompetenz der DEGUM-zertifizierten Ärzte wird bei der turnusmäßigen Rezertifizierung überprüft. Neben der eigenen
Weiterbildung sind DEGUM-Kursleiter der Stufen II und III auch verpflichtet, ihr Wissen und Können regelmäßig im Rahmen von DEGUM-zertifizierten Kursen weiterzugeben, um dieses hohe Niveau zu halten und auszubauen.

Eine Abteilung kann zertifiziert werden, wenn unter anderem mindestens ein DEGUM-Ausbilder (Stufe II) in der Abteilung arbeitet, und wenn ein Ausbildungscurriculum nachgewiesen wird. Die Zertifizierung ist 3 Jahre gültig und kann verlängert werden. Sobald eine Klinik über 3 DEGUM-zertifizierte Abteilungen verfügt, kann sie sich als Interdisziplinäres Ultraschall-Zentrum zertifizieren lassen. Wo es zertifizierte Abteilungen und Zentren gibt, erfahren Interessierte unter www.degum.de/service/ zertifizierte-abteilungen-zentren.html. 